\title{
A New Method for Characterizing 3D Microstructures Using Lab-based Diffraction Contrast Tomography
}

\author{
Hrishikesh Bale ${ }^{1}$, Will Harris ${ }^{1}$, Steve Kelly ${ }^{1}$, Nicolas Gueninchault ${ }^{2}$, Jun Sun $^{2}$, Erik Lauridsen ${ }^{2}$ \\ 1. Carl Zeiss X-ray Microscopy, Pleasanton, CA, USA. \\ 2. Xnovo Technology ApS, Køge, Denmark
}

While X-ray micro-tomography has been in use for some time both in synchrotron and laboratory-based architectures, it has traditionally operated under a single absorption-based contrast mechanism. In this operating mode, an X-ray projection image is formed due to spatially varying density within the sample, resulting in varying attenuation of the incident X-ray beam with reconstruction yielding a 3D map of sample density. This method has been extended by phase contrast methods, using either propagation based phase contrast, through the use of Talbot-Lau interferometer gratings, and Zernike arrangements, to better differentiate low-Z materials or multiple phases of very similar atomic density by enhancing the detection of differences in index of refraction at the boundaries of different phases.

However, neither approach can discern the crystallographic information within polycrystalline samples. Knowledge of the grain structure and crystallographic orientation distribution within polycrystalline samples is critical to the understanding of the fracture mechanisms that operate at the length scale of the grains. To address this problem, several years back, a few select synchrotron beamlines began developing methods to not only capture absorption/transmission information, but diffraction signals as well. $[1,2]$. Since then, the diffraction tomography technique has gained considerable traction over the years as a complementary method to EBSD due to its ability to uniquely obtain bulk, 3D grain structures in a non-destructive fashion, opening the door for large-scale grain structure analysis or time-dependent evolutionary studies. Due to the exclusive presence of the technique at the synchrotron however, its accessibility to the wider research community has been inherently limited.

Recently, the adaptation of the method to laboratory-based systems has broadened its user base and impact. In this work, a lab-based adaptation is presented, termed lab diffraction contrast tomography (LabDCT). The LabDCT imaging modality is implemented on the ZEISS Xradia Versa laboratory Xray microscope, and adapted to operate with a polychromatic divergent beam (as opposed to the typically collimated, monochromatic beam at synchrotron beamlines). Mounting a sample in a Laue focusing condition with an equidistant source-sample and sample-detector arrangement, individual grains satisfying the Bragg condition produce diffraction spots on a specialized high resolution detector. The polychromatic beam provides a unique advantage wherein a majority of the grains within a sample simultaneously satisfy the Bragg condition due to the wide spectrum of wavelengths. Rotation of the sample then yields a series of projections with diffraction patterns, which are reconstructed using a dedicated 3D grain reconstruction software (GrainMapper3D, Xnovo Technology ApS, Køge, Denmark) to yield crystallographic information including grain orientation, location of center of mass, and morphology for a large number of grains within the sample. This information can be utilized to complement microstructural data obtained by traditional absorption-based tomography such as voids, inclusions, or secondary non-crystalline phases.

This paper will present results demonstrating the latest developments in LabDCT by means of several applications. $[3,4]$ Included in the discussion will be highlights of the flexibility of the method, including 
capabilities to investigate cubic and hexagonal crystal symmetries, as well as both metallic and nonmetallic polycrystalline materials. In particular, examples will cover studies related to tracking 4D grain growth during annealing processes, microstructural impacts on corrosion behavior, and connections between grain boundary characteristics, inclusions, and performance in polycrystalline silicon for solar cell applications.

Lastly, we will consider future directions and developments of the LabDCT method, as well as possibilities of correlative coupling to related techniques for an enhanced understanding of materials structure evolution in 3D and across multiple length scales.

References:

[1] U. Lienert et al., Journal of the Minerals, Metals \& Materials Society, 63 (2011), p. 70.

[2] D. Juul Jensen and H.F. Poulsen, Materials Characterization, 72 (2012), p. 1.

[3] R. Keinan et al., Acta Materialia, In Press (2018).

[4] S. A. McDonald et al., Scientific Reports, 7 (2017), p. 5251.
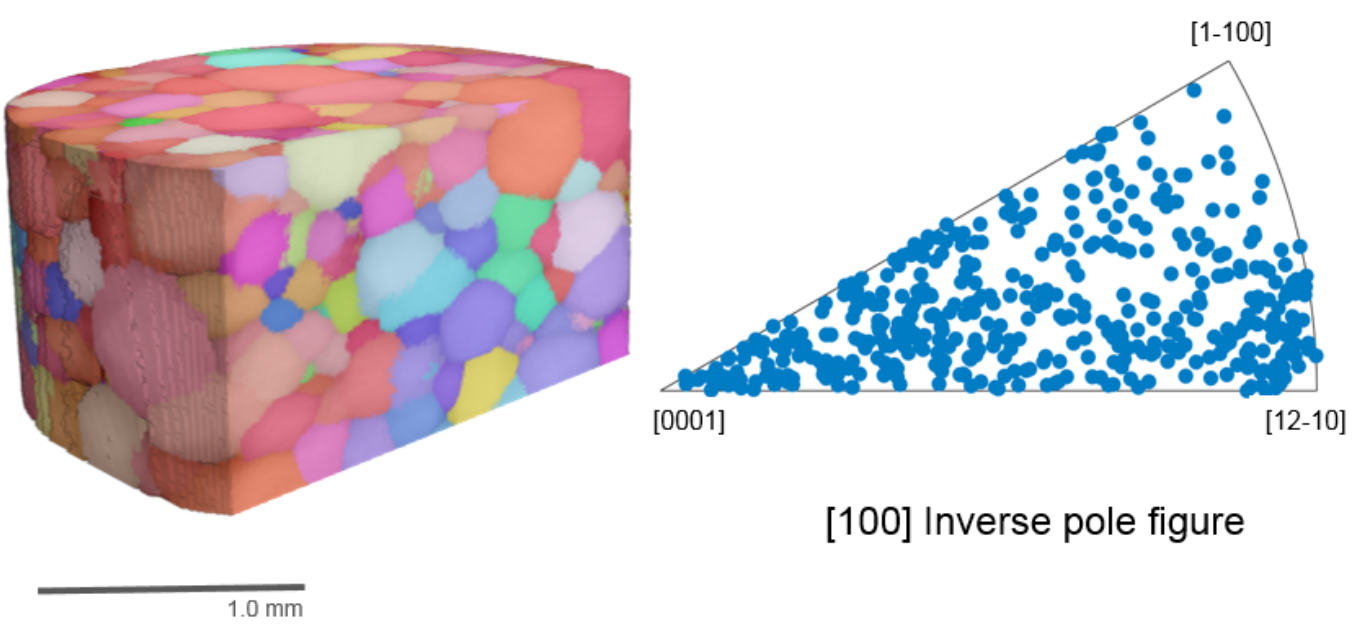

[100] Inverse pole figure

Figure 1. Titanium-7\%Al (hexagonal) results. Note the much larger volume size compared to typical EBSD analysis, particularly 3D EBSD, making LabDCT a strong complementary technique for sampling at larger length scales. Data can be mapped to an inverse pole figure (right) for textural analysis.

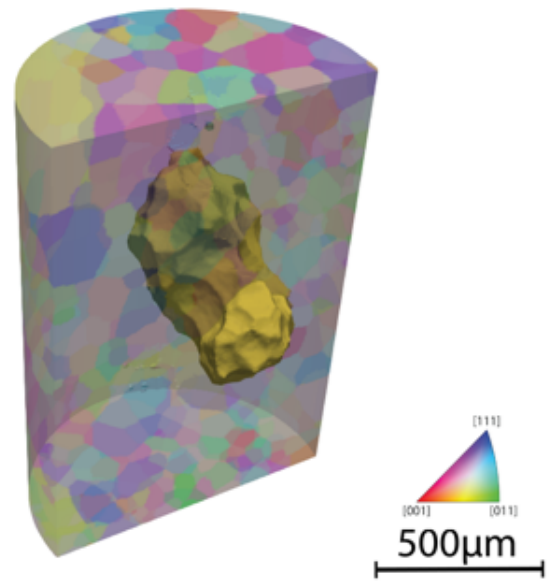

Figure 2. Abnormal grain growth detected in Armco iron. 